\title{
Editorial: Green New Deal!? Wie rot ist das neue Grün?
}

$V_{d}$ or dem Ausbruch der Corona-Pandemie hat die ökologische Krise einen enormen politischen Bedeutungszuwachs erfahren. So hat etwa die Präsidentin der Europäischen Kommission, Ursula von der Leyen, kurz nach ihrem Amtsantritt Ende 2019 den European Green Deal (EGD) als Schwerpunkt ihrer Amtszeit präsentiert. Die Corona-Krise hat den EGD nicht von der politischen Bildfläche verdrängt. Vielmehr präsentiert die Europäische Kommission diesen nun, angesichts der Corona-Pandemie modifiziert und durch den geplanten europäischen Wiederaufbaufonds mit neuer finanzieller Schlagkraft versehen, als zukunftsweisenden Ausweg aus der Krise: Mit dem Green Deal soll die EU bis zum Jahr 2050 klimaneutral werden. Jetzt sei »unsere Chance, unsere Volkswirtschaften anders zu gestalten und sie widerstandfähiger $\mathrm{zu}$ machen «, so von der Leyen in einer Videobotschaft im Mai 2020. Damit greift die Kommissionspräsidentin ein Thema auf, das dank sozialer Bewegungen wie Fridays for Future oder Extinction Rebellion vor der Corona-Krise stark in die öffentliche Aufmerksamkeit gerückt wurde: den Klimawandel. Die vorliegende PROKLA greift die jüngsten politischen Vorstöße auf, geht aber zugleich vielen grundlegenden Fragen nach, die mit dem Thema verbunden sind. Zugespitzt geht es um die Frage, ob sich der Kapitalismus in einer Weise verändert oder verändern lässt, die die Ursachen der ökologischen Katastrophen beseitigt kann.

Tobias Haas und Isabel Jürgens argumentieren in ihrem Einspruch in der vorliegenden Ausgabe, dass der EGD weitgehend in der Logik einer ökologischen Modernisierung verfangen bleibt, jedoch die Programmatik des EGD durchaus Anknüpfungspunkte für progressive Akteure liefert, die den Klimawandel politisieren. Simone Claar untersucht in ihrem Beitrag den EGD im Hinblick auf dessen Auswirkungen für Afrika. Sie zeigt anhand der Beispiele der erneuerbaren Energien und der Biodiversitätspolitik auf, dass der EGD darauf ausgerichtet ist, die bestehenden Abhängigkeitsmuster unter begrünten Vorzeichen zu erneuern.

Die Idee eines Deals, wie er etwa von der Europäischen Kommission aufgegriffen wird, ist keineswegs neu, sie hat einen historischen Vorläufer. Nach der Weltwirtschaftskrise 1929 wurde zunächst in den USA ein solcher praktiziert - und fand wenige Jahre später seine wissenschaftliche Legitimation im 
Keynesianismus, der weniger mit Keynes als mit der neuen Wirtschaftspolitik zu tun hatte, die sich nach 1945 in vielen industrialisierten Ländern etablierte: In Zeiten wirtschaftlicher Stagnation oder gar einer Krise soll der Staat als Wirtschaftsakteur stabilisierend auf Beschäftigungsniveau und Konjunktur einwirken, indem öffentliche Ausgaben ausgeweitet werden, schuldenfinanziert oder dank Abschöpfung hoher Privatvermögen und Einkommen. Die New-Deal-Idee hat demnach nicht nur eine wirtschafts- sondern immer auch eine sozialpolitische Dimension. Während der Keynesianismus allerdings verging, kamen die nächsten Krisen: nicht nur Wirtschaftskrisen, sondern auch eine ökologische Krise, die die Grundlagen des menschlichen Überlebens infrage stellt.

Vor diesem Hintergrund schlugen linke Grüne bereits Ende der 1980er Jahre einen Grünen New Deal vor. Zu Beginn der 1990er Jahre ging daraus die Idee für ein rot-rot-grünes Crossover-Projekt hervor: Durch die Verknüpfung der ökologischen mit der sozialen Frage sollte, so heißt es in einem einschlägigen Beitrag von Willi Brüggen (2001) im Historisch-kritischen Wörterbuch des Marxismus, »die Basis für ein längerfristig angelegtes strategisches Bündnis zwischen progressiven Sozialdemokraten, Sozialisten und Grünen gelegt werden.« Eine Wendung nahm die Debatte spätestens mit der Finanzkrise ab 2007. Das gesellschaftliche bzw. parteipolitische Bündnisprojekt mutierte $\mathrm{zu}$ einem eher technokratischen ökologischen Modernisierungsprogramm, das, so kritisierte Ulrich Brand (2009) in der
PROKLA 156, im Gegensatz zum rooseveltschen New Deal der 1930er Jahre weniger von einer progressiven Kräftekonstellation als von einem Technik- und Steuerungsoptimismus getragen wurde. Dem historischen New Deal widmet sich Steffen Lehndorff in seinem Einspruch und entfaltet das Argument, dass in der gegenwärtigen Krisenkonstellation progressive Akteure dringend an die Erfahrungen des New Deal anknüpfen und sich der Frage öffnen sollten, wie ein Green New Deal heute umgesetzt werden könnte. Christine Schickert geht in ihrem Beitrag der Frage nach, wie ein Green New Deal im regionalen Kontext umgesetzt werden könnte bzw. argumentiert, dass nur eine lokale und regionale Verankerung, die Veränderungsprozesse für die breite Bevölkerung erfahrbar und konkrete Projekte plausibel mache, die gesellschaftliche Voraussetzung dafür sei, einen gesamtgesellschaftlichen Green New Deal überhaupt umund durchzusetzen - als ein breit getragenes Projekt.

Gleich drei Beiträge gehen sehr grundlegenden Fragen nach und formulieren eine deutliche Skepsis gegenüber der Idee eines Green New Deal. Christian Zeller versteht die Idee eines Green New Deal als eine Quadratur des Kreises. Basierend auf der Analyse verschiedener GND-Konzepte argumentiert er, dass diese nicht mit dem kapitalistischen Wachstumszwang brechen und somit keine Antwort auf die sozial-ökologischen Krisen. Eine ähnliche Position vertritt Tone Smith, die in ihrem Beitrag ebenfalls mehrere Vorschläge vorstellt und kritisch diskutiert. Sie plädiert dafür, den Begriff New Deal gleich fallen 
$\mathrm{zu}$ lassen, weil mit ihm die Idee von keynesianischem Produktivismus und die vermeintliche Notwendigkeit von Wirtschaftswachstum verbunden sei. Vor dem Hintergrund der Vielfalt an Vorschlägen, die durchaus auch progressive Elemente aufweisen, problematisieren auch Ronja Morgenthaler und Lasse Thiele einerseits die Wachstumsorientierung auch progressiver GNDKonzepte, gehen jedoch andererseits der Frage nach, welche Ansatzpunkte es dennoch gibt, um diese Ansätze in eine post-kapitalistische Richtung weiter zu entwickeln. Dabei nehmen sie Bezug auf Eric Ohlin Wrights dreiteilige Transformationsstrategie. Meike Spitzner kritisiert die GND-Konzepte aus einer Suffizienzperspektive: Ihre These ist, dass es unverzichtbar sei, im Transformationskonzept selbst die strukturellen Ursachen von gesellschaftlichen Ungleichheiten geschlechtergerechtigkeitswirksam zum Thema zu machen. Am Beispiel der Verkehrswende zeigt sie, dass dies die Voraussetzung dafür ist, ökonomisch-sozial-ökologische Zusammenhänge zu erkennen und die Klima-, Ressourcen- und ökonomischen Krisen entsprechend politisieren zu können.

Während sich die letztgenannten Beiträge generell dem GND-Debattenfeld widmen, fokussiert Ingo Stützle in seinem Beitrag einen spezifischen, aber grundlegenden Aspekt der Ansätze, nämlich die Finanzierung und geldpolitische Flankierung eines GND. Innerhalb der GND-Debatten und angesichts der Folgekosten, die die globale Pandemie zeitigt, wird das Konzept der Modern Monetary Theory (MMT) intensiv diskutiert. In seinem Beitrag kritisiert Stützle die MMT-These, dass die
Finanzierung - etwa eines Green New Deal - kein Problem sei. Das mangelhafte Geld- und Kapitalismusverständnis verleite dazu, gesellschaftliche Kräfteverhältnisse auszublenden, weil die gesellschaftlichen Formen ignoriert werden, in denen sich der gesellschaftliche Reproduktionsprozess vollziehe: Geld, Waren - Steuern, öffentliche Güter.

Dass eine drohende ökologische Krise eine gesamtgesellschaftliche Herausforderung darstellt, haben inzwischen selbst Konservative verstanden - für die extreme Rechte stellt diese Konstellation eine ganz besondere Herausforderung dar. Alexander Neupert-Doppler beleuchtet deshalb nicht die Konzeption des GND, sondern analysiert aus einer ideologiekritischen Perspektive die Reaktion der »klimaskeptischen « AfD auf die Vorschläge eines Green Deals. Er argumentiert mit Karl Mannheim, dass die AfD das Konzept der Ökodiktatur als eine Form der Gegendystopie in der politischen Auseinandersetzung platziere, die gegen die reale Dystopie einer weiteren Eskalation der Klimakrise heraufbeschworen wird.

Im November 2020 fand die Mitgliederversammlung der Vereinigung zur Kritik der politischen Ökonomie 2020 statt - wie derzeit so vieles nur digital. Kurz darauf erhielten wir die sehr traurige Nachricht, dass am 14 . November 2020 überraschend Ulf Kadritzke verstarb. Er war der PROKLA als Autor und Mitglied des Redaktionsbeirats viele Jahre eng verbunden - und auf der letzten Mitgliederversammlung noch engagiert dabei. Mit seinen Ideen und seinem Engagement hat er nicht nur die PROKLA 
bereichert und geprägt. »Träumt weiter, aber mit offenen Augen « titelt der Nachruf von Rudi Schmidt in der vorliegenden Ausgabe - einem Aufruf, dem wir nachkommen werden. In Zukunft leider ohne Ulf.

Das Jahr 2021 ist ein historisches - wir feiern 50 Jahre PROKLA. Es ist einiges geplant. Nicht absehbar ist, ob wir die Vorhaben angesichts der widrigen Umstände alle realisieren können.
Vielleicht schaut ihr mal bei www.prokla.de vorbei oder abonniert unseren Newsletter.

\section{Literatur}

Brand, Ulrich (2009): Schillernd und technokratisch: Grüner New Deal als magic bullet in der Krise des neoliberal-imperialen Kapitalismus? In: PROKLA 156 39(3): 475-481. DOI: https://doi.org/10.32387/ prokla.v39i156.426.

Brüggen, Willi (1991): Grüner New Deal. In: Historisch-kritisches Wörterbuch des Marxismus, Band 5. Hamburg: 1062-1070.

\section{Der PROKLA Förderverein}

Die PROKLA erscheint seit 1971 und bietet politisch engagierte sozialwissenschaftliche und ökonomische Analysen. Allein von den Verkaufserlösen kann sich die PROKLA nicht finanzieren, und in die Abhängigkeit von Parteien oder großen Verlagen wollte sie sich nie begeben. Deshalb wird die PROKLA von einem Förderverein herausgegeben, der »Vereinigung zur Kritik der politischen Ökonomie e.V.», die jährlich in ihrer Vollversammlung die Redaktion der Zeitschrift wählt und die nächsten Themenschwerpunkte diskutiert.

Kritische Sozialwissenschaft kann nicht dem Markt überlassen werden. Ohne solidarische Strukturen und finanzielle Unterstützung sind Zeitschriften wie die PROKLA kaum möglich. Die finanziellen Beiträge der Vereinsmitglieder ermöglichen das Erscheinen der PROKLA, sie schaffen die Voraussetzungen für Kontinuität und Planbarkeit, wie sie für die Redaktionsarbeit unabdingbar sind. Wir freuen uns über weitere Mitglieder, regelmäßige Spenden oder einmalige Zuwendungen. Weitere Informationen teilen wir gerne per E-Mail mit (redaktion@prokla.de).

Vereinigung zur Kritik der politischen Ökonomie e.V. | Postbank Berlin | IBAN: DE17 100100100538135100 | BIC: PBNKDEFF 